Supporting Information

\title{
Accelerated Growth of Electrically Isolated Lithium Metal During Battery Cycling
}

Yibo Zhaot, Yifan Wuł, Huihui Liu, Sung-Liang Chen, and Shou-Hang Bo*

University of Michigan-Shanghai Jiao Tong University Joint Institute, Shanghai Jiao

Tong University, Shanghai, 200240, China.

*Corresponding author: shouhang.bo@sjtu.edu.cn 


\section{Table of contents}

Electrochemistry (Figure S1-S4)

Gas Chromatography Measurements (Table S1, Figure S5)

Photoacoustic Imaging (Figure S6-S10)

Electrochemical Impedance Spectroscopy Tests (Figure S11, Table S2 and Table S3)

\section{Electrochemistry}

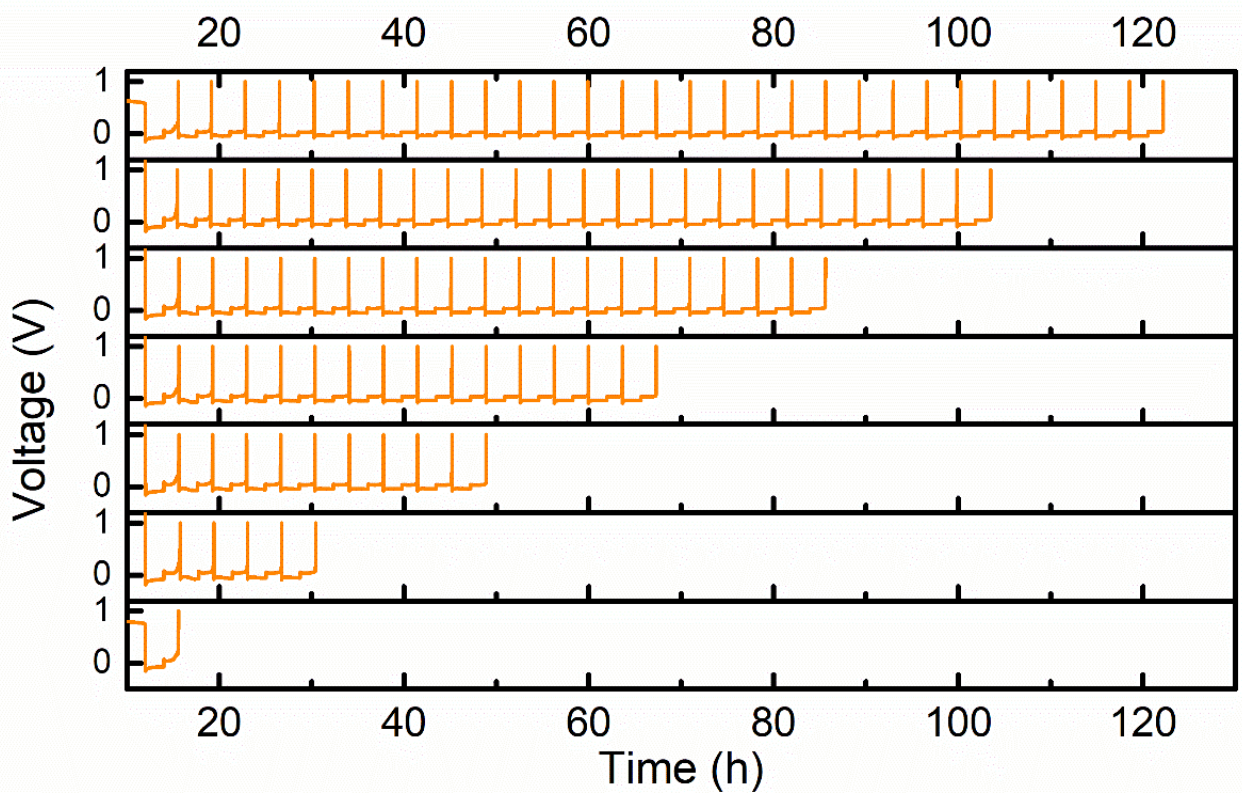

Figure S1. Voltage profile versus time for the cells that are cycled for 1, 5, 10, 15, 20,

25 , and 30 cycles (from bottom to top) under a current of $0.196 \mathrm{~mA}$ and discharge time of 2 hours for each cycle. The cut-off voltages were set to $-1 \mathrm{~V}$ and $1 \mathrm{~V}$. 


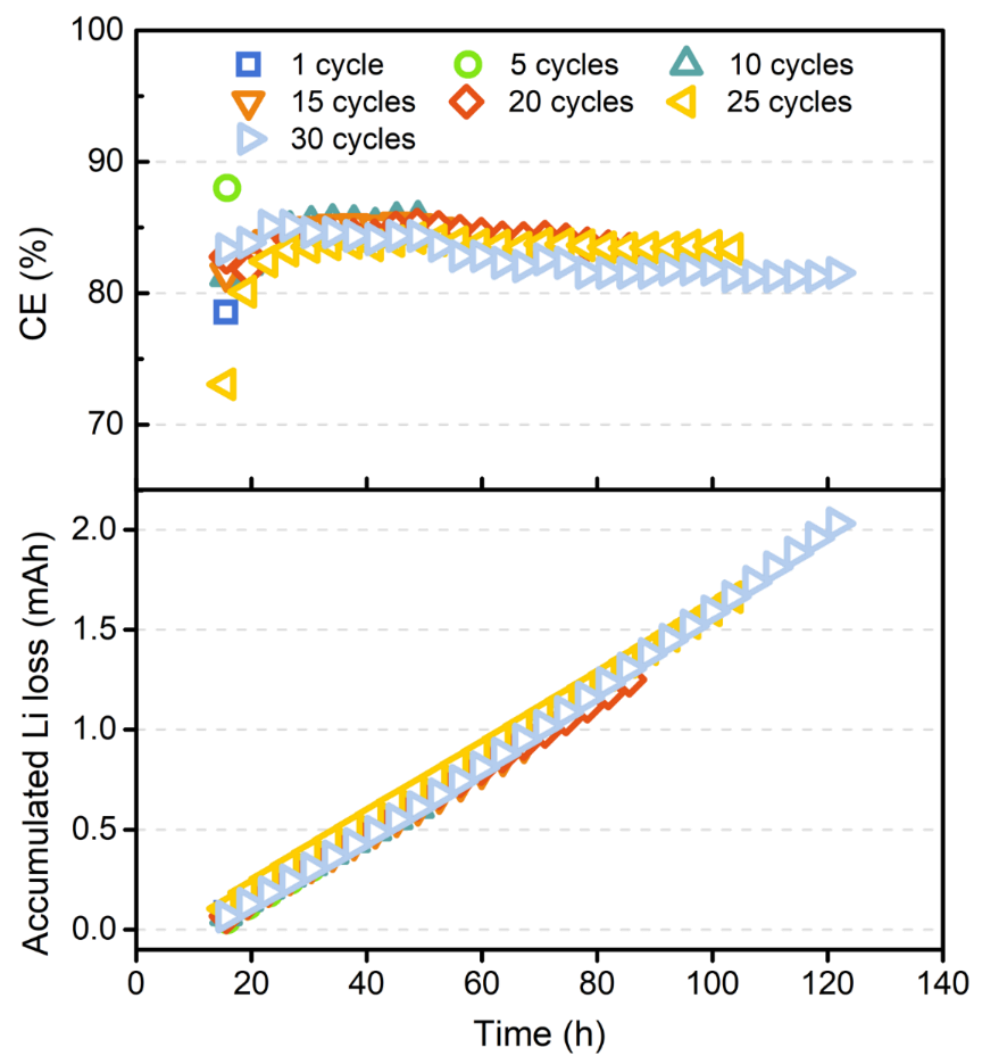

Figure S2. Coulombic efficiency (top figure) of the cells cycled for 1, 5, 10, 15, 20, 25, and 30 times, with respect to time. The Coulombic efficiency change tendencies are similar for all the cells apart from the first cycle. Accumulated Li loss records the total Li loss in $\mathrm{mA} \mathrm{h}$ after each cycle and is plotted with respect to time (bottom figure). 


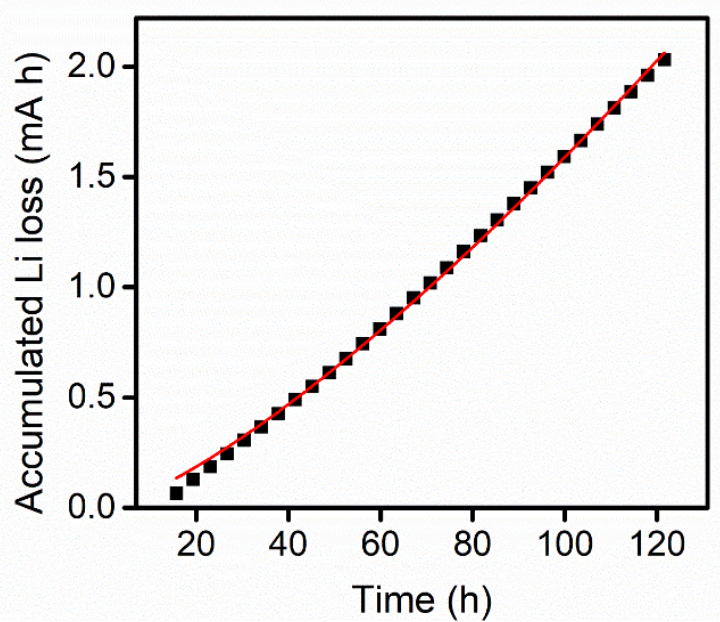

Figure S3. Power function $\left(\mathrm{y}=a \mathrm{x}^{\beta}\right)$ fitting for the accumulated Li loss of the 30-cycle cell. $\mathrm{R}^{2}=0.99854$. The fitting parameters are $a=0.00344$ and $\beta=1.3321$. 

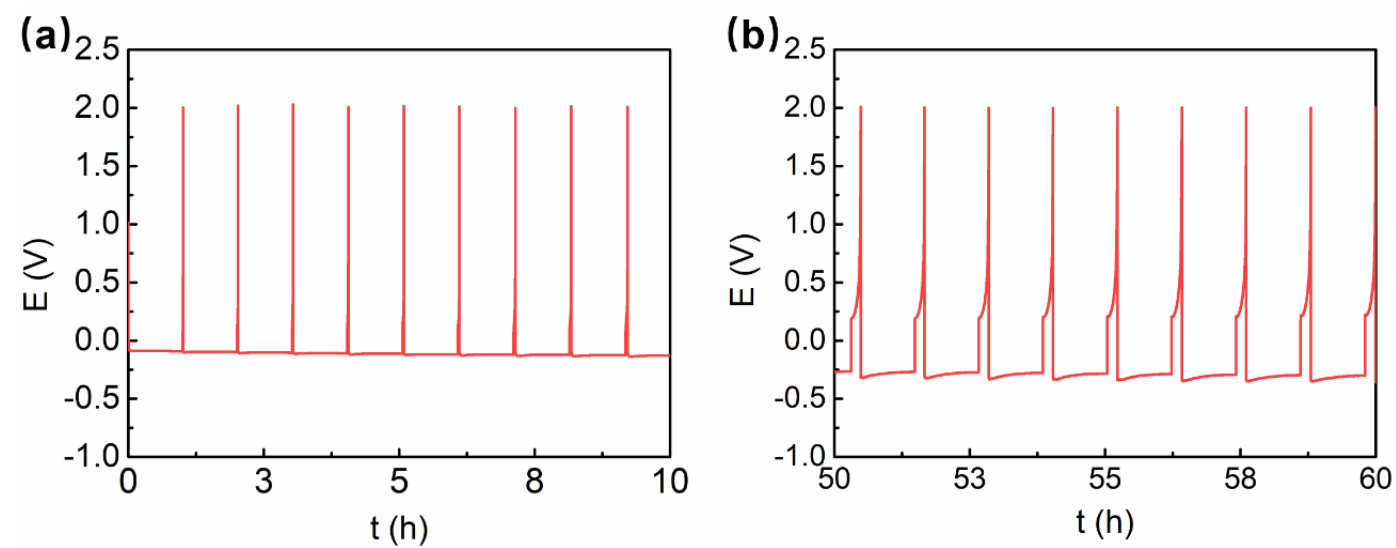

Figure S4. Cycling behavior of the Li/LGPS/Cu cell in (a) first few cycles and (b) extended cycles. 


\section{Gas Chromatography measurements}

Table S1: GC measurements and calculated $\mathrm{Li}^{0}$ amount

\begin{tabular}{c|ccc|ccc}
\hline & \multicolumn{3}{|c|}{$\mathrm{Li}^{0}$ on positive Cu current } & \multicolumn{3}{c}{$\mathrm{Li}^{0}$ on/in separators } \\
\hline $\begin{array}{c}\text { Cycle } \\
\text { numbers }\end{array}$ & $\begin{array}{c}\text { Conc. } \\
(\%)\end{array}$ & $\begin{array}{c}V \\
(\mathrm{~mL})\end{array}$ & $\begin{array}{c}\mathrm{Li}^{0} \\
(\mu \mathrm{mol})\end{array}$ & $\begin{array}{c}\text { Conc. } \\
(\%)\end{array}$ & $\begin{array}{c}V \\
(\mathrm{~mL})\end{array}$ & $\begin{array}{c}\mathrm{Li}^{0} \\
(\mu \mathrm{mol})\end{array}$ \\
\hline 1 & 0.042 & 72.76 & 2.5465 & 0.000 & 71.81 & 0 \\
5 & 0.161 & 70.03 & 8.8099 & 0.001 & 70.27 & 0.0586 \\
10 & 0.308 & 71.10 & 17.644 & 0.003 & 74.90 & 0.1872 \\
15 & 0.481 & 71.71 & 27.459 & 0.007 & 75.16 & 0.1998 \\
20 & 0.678 & 70.73 & 38.119 & 0.006 & 72.05 & 0.1687 \\
25 & 0.969 & 63.37 & 50.996 & 0.008 & 72.02 & 0.9602 \\
30 & 0.563 & 133.68 & 62.931 & 0.004 & 129.57 & 0.7558 \\
\hline
\end{tabular}

From each measurement, a Conc. value was obtained by comparing the peak area from the $\mathrm{H}_{2}$ gas inside the injected 1-mL gas and the calibration curve of standard $\mathrm{H}_{2}$ gas. The calibration curve is a linear fitting to correlate peak area of $\mathrm{H}_{2}$ with the gas concentration. Thus, the value of Conc. also represents the concentration of $\mathrm{H}_{2}$ inside the flask, as shown in Table S1. The volumes of the flasks were measured by measuring the mass of water with same volume. The mass of water was then converted to flask volume using the density of water as $1 \mathrm{~g} \mathrm{~cm}^{-3}$ in Table $\mathrm{S} 1$. The calculation of $\mathrm{Li}^{0}$ amount then follows the equation

$$
n_{L i}=2 n_{H_{2}}=2 \times \frac{p V}{R T}=2 \times \frac{1.015 \times 10^{5} \times V}{8.31 \times 298.15}
$$

where $p$ represents ambient air pressure, $V$ is the volume of $\mathrm{H}_{2}, R$ is ideal gas constant and $T$ is the temperature. 


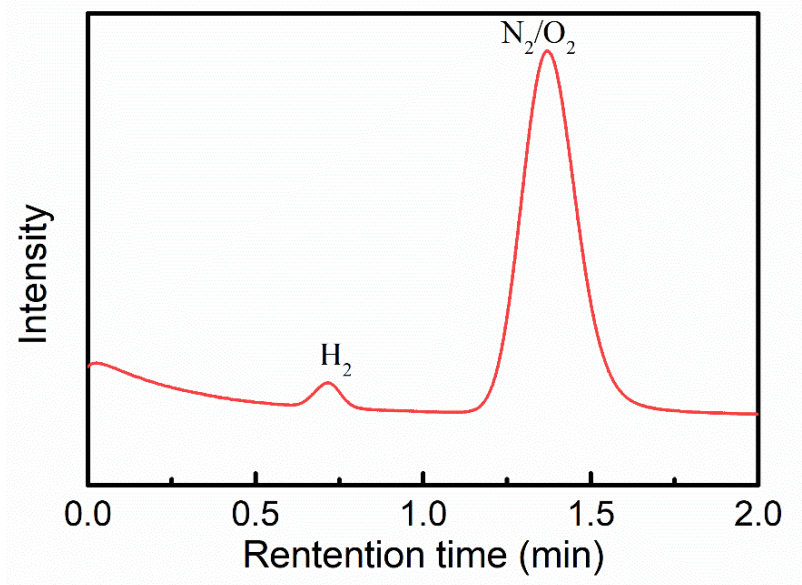

Figure S5. GC profile by water titration on $\mathrm{Cu}$ current collector of a 1-cycle sulfide solid electrolyte $\mathrm{Li} / \mathrm{Cu}$ cell. 


\section{Photoacoustic imaging}

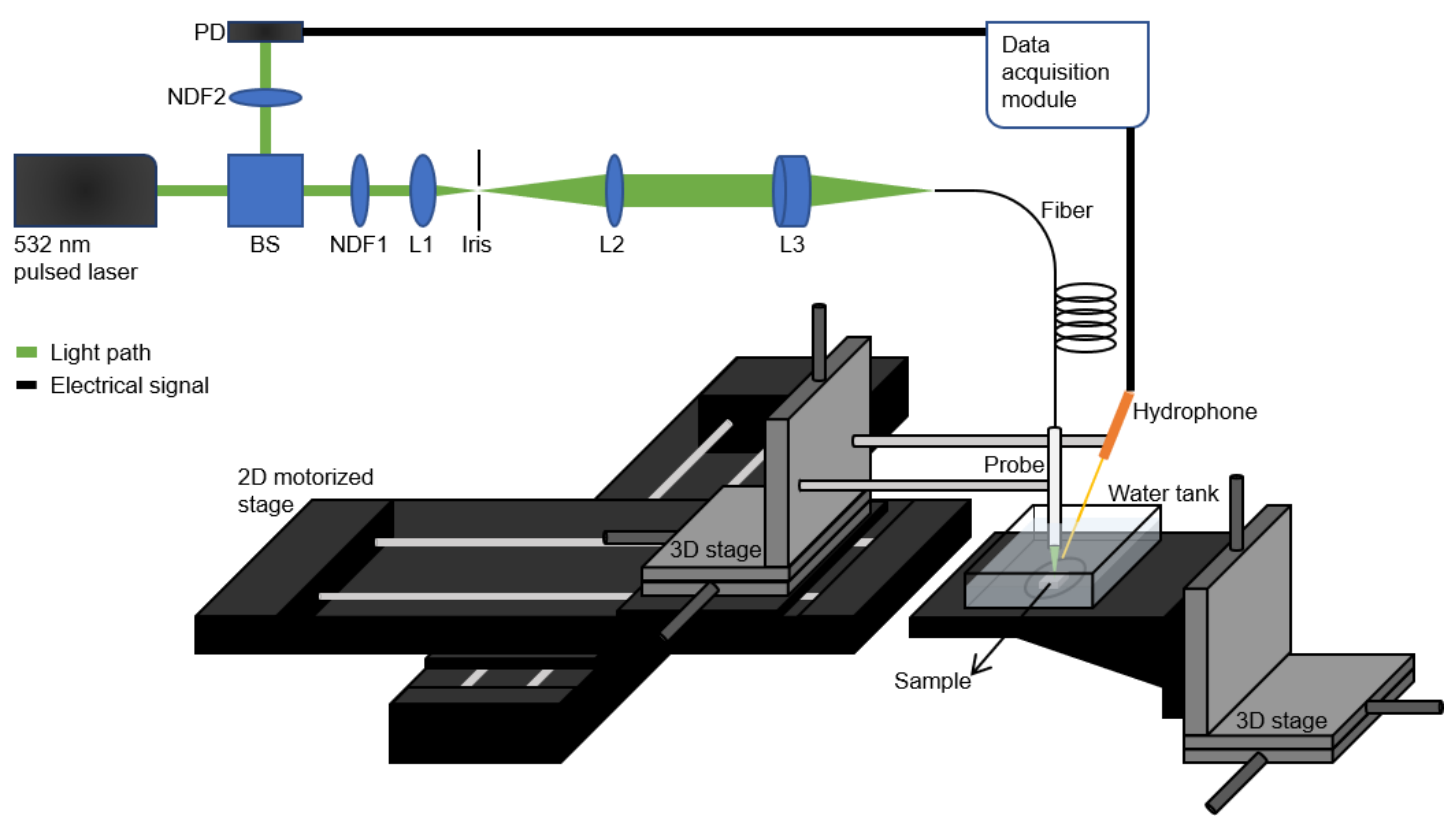

Figure S6. Schematic of the probe-based PA microscope system. BS, beamsplitter;

NDF1\&2, neutral density filters; PD, photodiode; L1\&L2, plano-convex lenses; L3, doublet lens.

Figure S6 shows the schematic of the home-built probe-based PA microscope used in this work. The setup of this PA microscope was mostly the same as reported in our previous work. Light from a $532 \mathrm{~nm}$ pulsed laser source (FDSS532-Q4, CryLaS) with the repetition frequency of $1 \mathrm{kHz}$ and the pulse width $\leq 1.3 \mathrm{~ns}$ was split into two paths by a 9:1 beamsplitter (BS025, Thorlabs). Ten percent of the light was reflected, attenuated by a neutral density filter, and then detected by a photodiode (DET10A2, Thorlabs) to provide trigger signals for data acquisition. The rest of the light was transmitted, attenuated also by a neutral density filter, and then expanded by a beamshaping path consisting of an iris (CP20S, Thorlabs) and two plano-convex lenses (GCL-0101, Daheng Optics). After that, the collimated beam was coupled into 
an optical fiber (SMF-28e, Connet, Shanghai) by a doublet lens (GCL-0106, Daheng Optics). Finally, the light coming out of the fiber was focused on one spot at a time by a plano-convex lens (GCL-010130, Daheng Optics) to excite PA waves. 


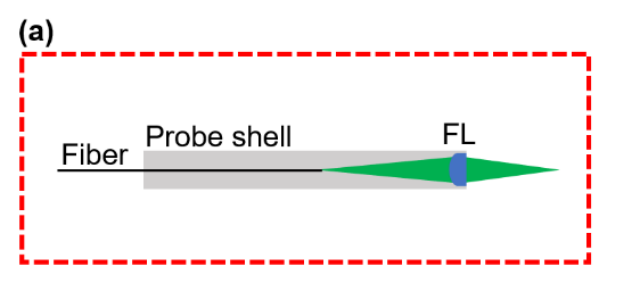

(b)

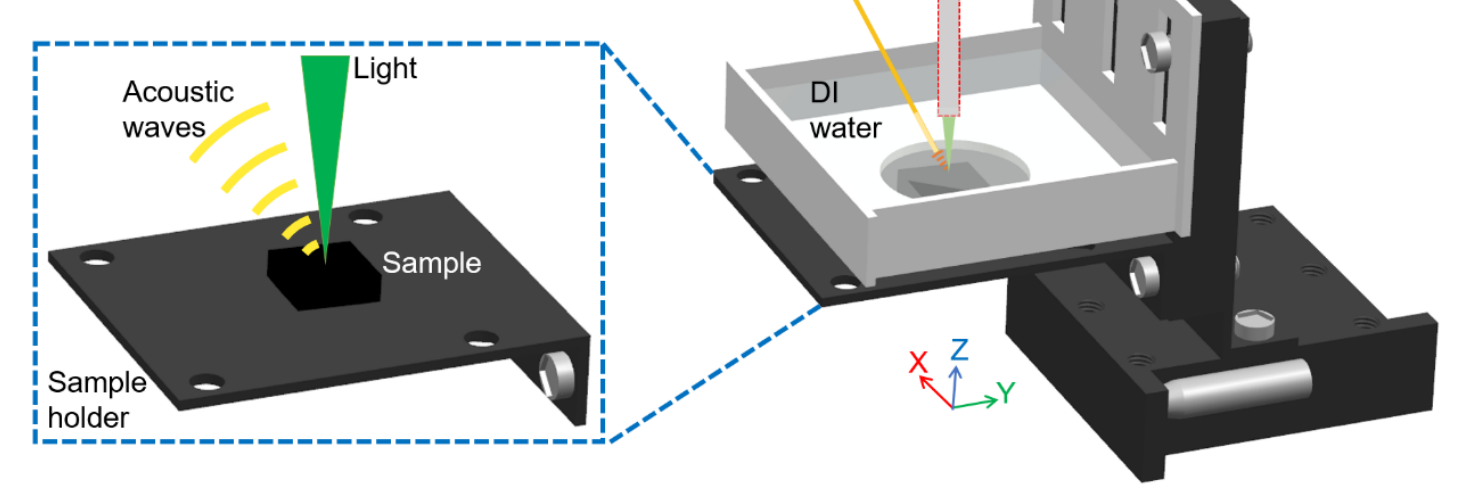

Figure S7. (a) Schematic of the probe; (b) Zoomed-in figure for the sample part of the

PA microscope. FL, focusing lens.

The excited PA waves from one spot were detected by a needle hydrophone with the central frequency of $35 \mathrm{MHz}$ and transformed into analog electrical signals, which were then amplified by a pre-amplifier (ZFL-500LN-BNC+, Mini-Circuits, New York) and eventually acquired as an A-line by a digitizer (CSE1422, GaGe, Illinois) with the sampling frequency of $200 \mathrm{MS} \mathrm{s}^{-1}$. Images of the sample were obtained by using a twodimensional (2D) motorized stage (Physik Instrumente [PI], Karlsruhe, Germany) for raster scanning of the probe and the hydrophone together in the horizontal (XY) plane and combining all A-lines. Note that to avoid signal attenuation during acoustic wave propagation, coupling agents are essential. Therefore, the end of the needle hydrophone was immersed in de-ionized (DI) water. At the bottom of the water tank, an imaging window covered by a polyethylene (PE) membrane was created. These are also shown in Figure S7(b), which presents a zoomed-in figure for the sample part in Figure S6. 
During PA imaging experiments, ultrasonic gels were added between the sample and the PE membrane as coupling agents. 


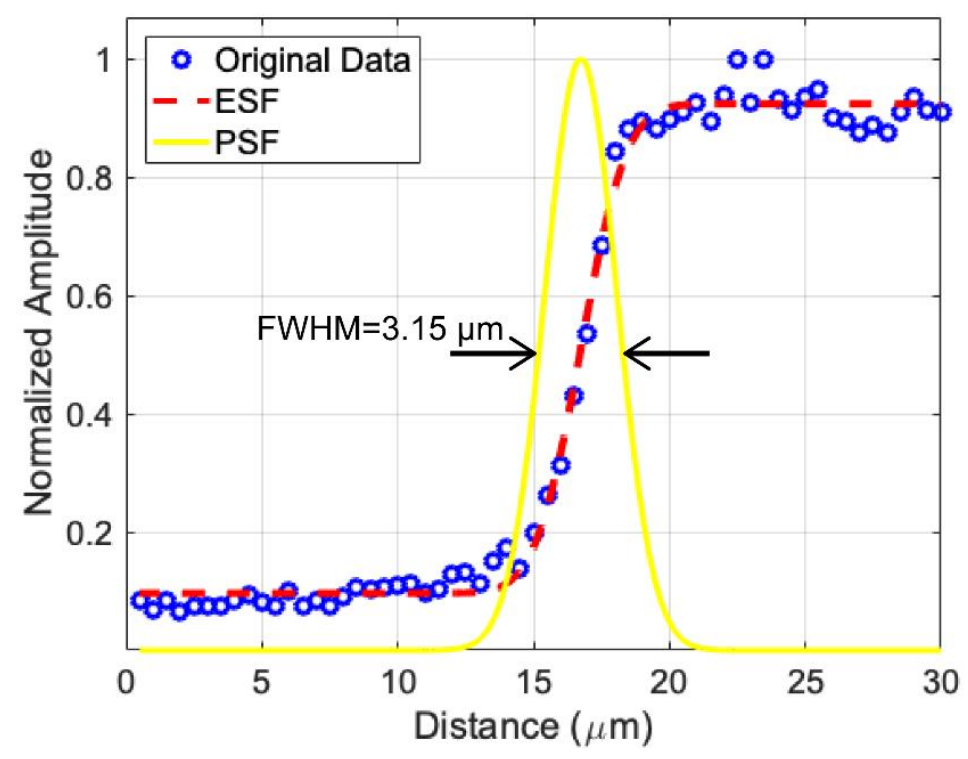

Figure S8. Lateral resolution of the PA microscope.

The lateral resolution of this system was calibrated by scanning across a razor blade edge and fitting the PA intensity profile for an edge spread function (ESF), whose first derivatives denoted the point spread function (PSF) of the system. The lateral resolution of the system was estimated as the full width at half maximum (FWHM) of PSF and calculated to be $\sim 3.15 \mu \mathrm{m}$. 


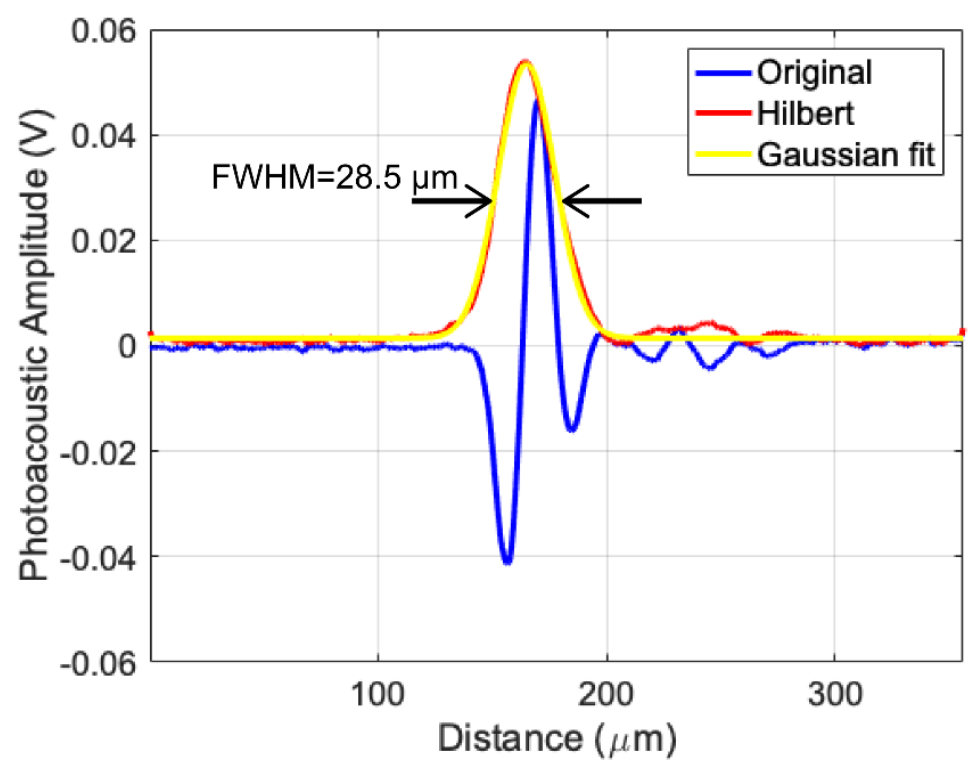

Figure S9. Axial resolution of the PA microscope.

The axial resolution of this system was calibrated by taking an A-line from a 6- $\mu \mathrm{m}-$ diameter carbon fiber and applying Hilbert transform. The FWHM of the signal envelope gave the axial resolution of the system as $\sim 28.5 \mu \mathrm{m}$. 

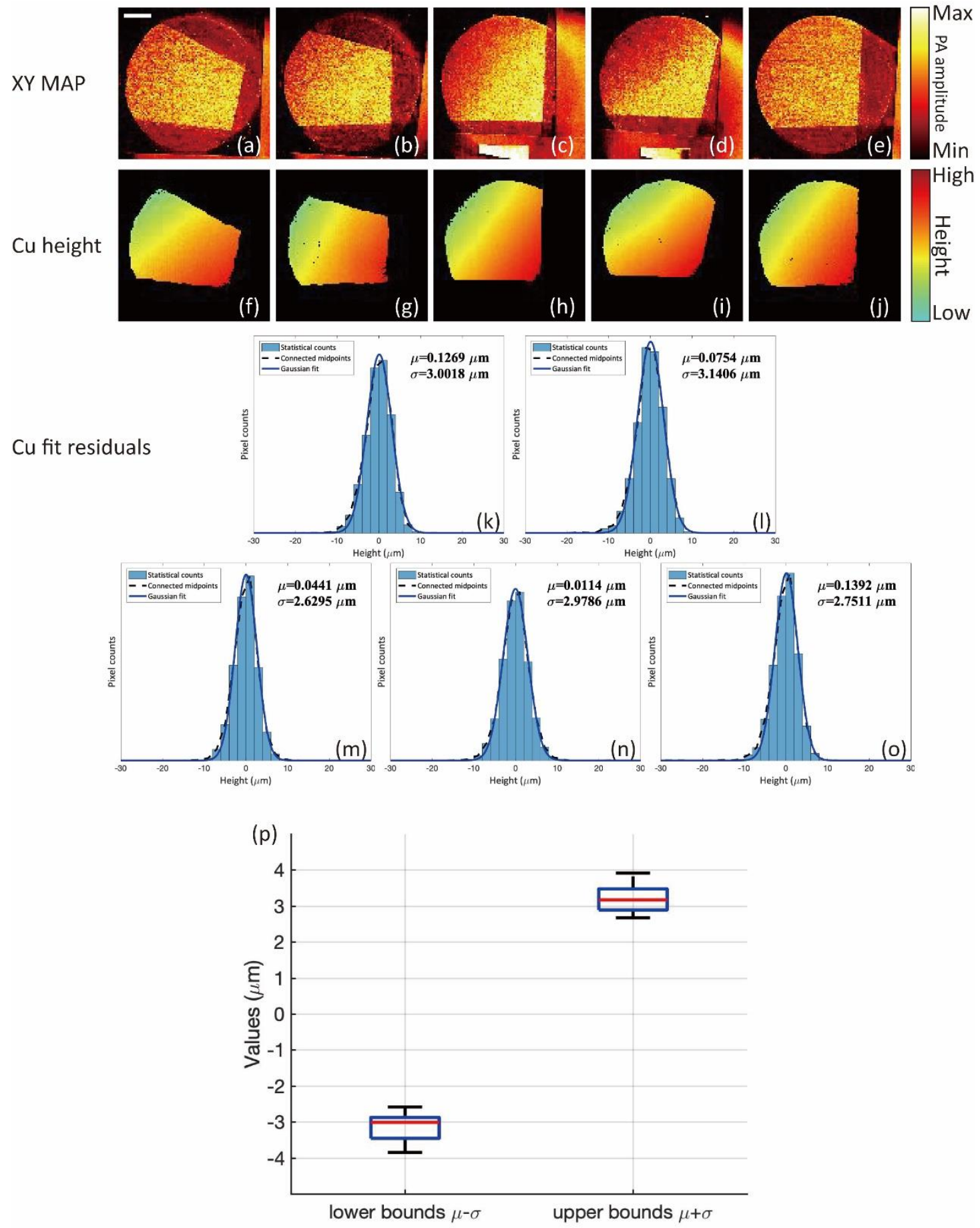

Figure S10. (a-e) PA XY MAP images and (f-j) height-encoded maps of five pristine $\mathrm{Cu}$ pellets' surfaces, respectively. (k-o) Histograms of residuals between the PA-imaged height and the corresponding fitted height for five pristine $\mathrm{Cu}$ pellets, respectively. Blue lines are Gaussian profiles fitted to the residual distributions; $\mu$ and $\sigma$ are mean and standard deviation values extracted from each Gaussian profile (Scale bar: $2 \mathrm{~mm}$ ). (p) 
Box plots for interval boundaries $\mu \pm \sigma$ for all examined pristine $\mathrm{Cu}$ pellets.

A sampling inspection method was exploited to derive variations in the surface height of the $\mathrm{Cu}$ current collector used in this manuscript from those of some other pristine $\mathrm{Cu}$ pellets. To ensure the reliability of this derivation, we took multiple pristine $\mathrm{Cu}$ pellets with the same product type and specifications as the $\mathrm{Cu}$ current collector and processed them by the same procedure of preparing the current collector.

Variations in the surface height of pristine $\mathrm{Cu}$ pellets were estimated as follows. We first conducted PA imaging on these pellets to obtain height-encoded maps of their surfaces. Polynomial fits were then applied to every height-encoded map; thus, variations in the PA-imaged surface height of each $\mathrm{Cu}$ pellet could be represented by the residuals between each height-encoded map and the corresponding fitted smooth surface. Figure S10 (a-e) and (f-j) show the PA XY MAP images and height-encoded maps of five samples among all examined $\mathrm{Cu}$ pellets. The histograms of their residuals are presented in Figure S10(k-o). Plotting histograms of residuals for all $\mathrm{Cu}$ pellets revealed that variations in the PA-imaged surface height of a pristine $\mathrm{Cu}$ pellet generally follow a Gaussian distribution, as shown in Figure S10(k-o). Therefore, the probability of a variation in the PA-imaged surface height of a pristine $\mathrm{Cu}$ pellet within the interval $(\mu-\sigma, \mu+\sigma)$ is $\sim 0.683$, where $\mu$ and $\sigma$ are mean and standard deviation values extracted from the Gaussian profile fitted to the corresponding residual distribution. Deriving from the statistical analysis on the interval boundaries for all pristine $\mathrm{Cu}$ 
pellets, as shown in Figure S10(p), we consider the majority of variations in the surface height of the $\mathrm{Cu}$ current collector are also within the range of $\pm 4 \mu \mathrm{m}$. This range is quite minor compared with the average height $(\sim 39.76 \mu \mathrm{m})$ of "dead" Li. Thus, in this case, variations in the surface height of the $\mathrm{Cu}$ current collector would not cause significant effects on the height of "dead" Li. 


\section{Electrochemical Impedance Spectroscopy Tests}

Table S2: EIS fitting parameters for Figure $4 \mathrm{~b}$ of the main text.

\begin{tabular}{cccccc}
\hline $\mathrm{t}$ & $\mathrm{R} 1$ & Error & $\mathrm{R} 2$ & Error & $\mathrm{R}$ \\
$(\mathrm{h})$ & $(\Omega)$ & $(\Omega)$ & $(\Omega)$ & $(\Omega)$ & $(\Omega)$ \\
\hline 0 & 6.627 & 0.80961 & 113.9 & 1.4132 & 107.273 \\
6.06 & 21.16 & 0.58937 & 166.7 & 1.076 & 145.54 \\
12.07 & 20.6 & 0.63214 & 188.8 & 1.1086 & 168.2 \\
68.99 & 9.921 & 0.62224 & 296.1 & 0.8821 & 286.179 \\
81.06 & 8.986 & 0.40448 & 289.9 & 0.96254 & 280.914 \\
93.14 & 8.777 & 0.53327 & 300.9 & 1.0501 & 292.123 \\
105.22 & 8.789 & 0.42732 & 313.3 & 0.86981 & 304.511 \\
\hline
\end{tabular}


(a)

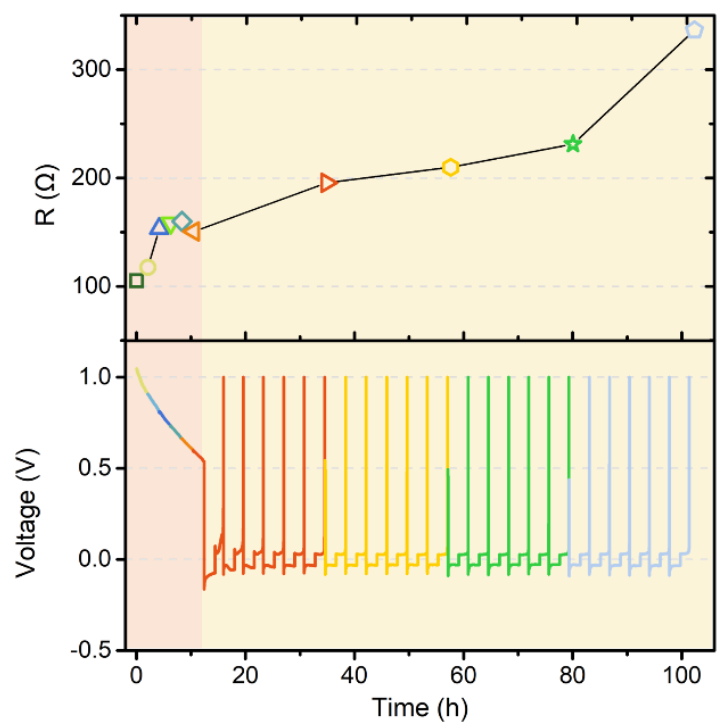

(b)

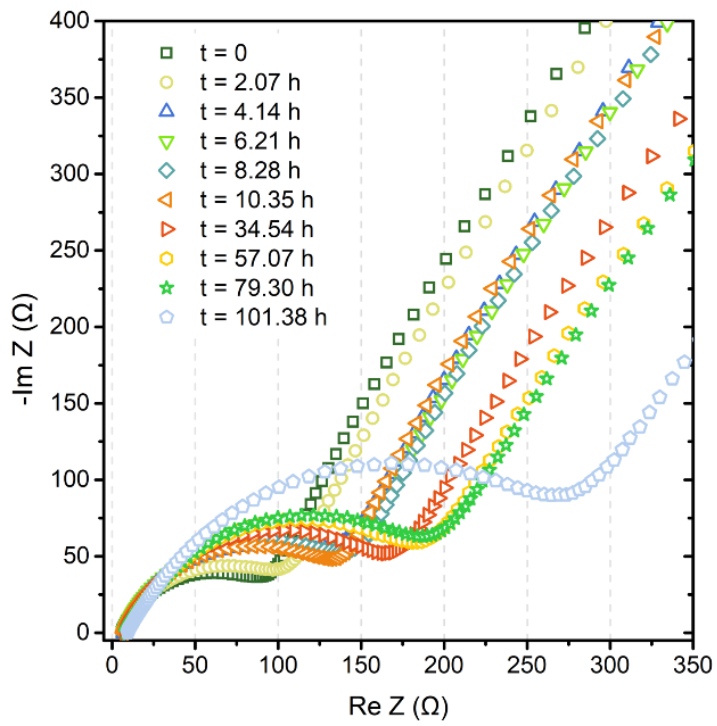

Figure S11. (a) Calculated resistance with respect to time during 10-hour's rest (pink region) and 26 cycles under $0.196 \mathrm{~mA}$ (yellow region). EIS data are shown in (b). 
Table S3. EIS fitting parameters for Figure S11.

\begin{tabular}{cccccc}
$\mathrm{t}$ & $\mathrm{R} 1$ & $\begin{array}{c}\text { Error } \\
(\Omega)\end{array}$ & $\begin{array}{c}\mathrm{R} 2 \\
(\Omega)\end{array}$ & $\begin{array}{c}\text { Error } \\
(\Omega)\end{array}$ & $\begin{array}{c}\mathrm{R} \\
(\Omega)\end{array}$ \\
\hline 0 & 5.677 & 0.73424 & 112 & 1.1141 & 106.323 \\
2.07 & 6.878 & 0.68495 & 126.6 & 1.0975 & 119.722 \\
4.14 & 6.862 & 0.66229 & 163.3 & 1.0894 & 156.438 \\
6.21 & 6.824 & 0.68385 & 168.4 & 1.0508 & 161.576 \\
8.28 & 6.9 & 0.63759 & 170.5 & 0.94991 & 163.6 \\
10.35 & 6.883 & 0.62851 & 160.5 & 0.84822 & 153.617 \\
34.54 & 7.283 & 0.5264 & 200.1 & 0.75782 & 192.817 \\
57.07 & 8.464 & 2.7619 & 221.7 & 0.90122 & 213.236 \\
79.30 & 8.206 & 0.45785 & 234.4 & 0.78016 & 226.194 \\
101.38 & 9.297 & 0.40096 & 338.6 & 0.63517 & 329.303 \\
\hline
\end{tabular}

